\title{
Preparation and Optimization of 10-Hydroxycamptothecin Nanocolloidal Particles Using Antisolvent Method Combined with High Pressure Homogenization
}

\author{
Bolin Lian, ${ }^{1}$ Yong Li, ${ }^{2}$ Xiuhua Zhao, ${ }^{1}$ Yuangang $\mathrm{Zu},{ }^{1}$ Ying Wang, \\ Yin Zhang, ${ }^{1}$ and Yuanyuan $\mathrm{Li}^{1}$ \\ ${ }^{1}$ Key Laboratory of Forest Plant Ecology, Northeast Forestry University, Ministry of Education, Harbin, Heilongjiang 150040, China \\ ${ }^{2}$ School of Food Science and Technology, National Engineering Research Center of Seafood, Dalian Polytechnic University, \\ Dalian, Liaoning 116034, China \\ Correspondence should be addressed to Xiuhua Zhao; xiuhuazhao@nefu.edu.cn and Yuangang Zu; yuangangzu@163.com
}

Received 27 February 2017; Accepted 20 April 2017; Published 24 May 2017

Academic Editor: José L. Arias Mediano

Copyright $\odot 2017$ Bolin Lian et al. This is an open access article distributed under the Creative Commons Attribution License, which permits unrestricted use, distribution, and reproduction in any medium, provided the original work is properly cited.

\begin{abstract}
The aim of this study was to prepare 10-hydroxycamptothecin nanocolloidal particles (HCPTNPs) to increase the solubility of drugs, reduce the toxicity, improve the stability of the drug, and so forth. HCPTNPs was prepared by antisolvent precipitation (AP) method combined with high pressure homogenization (HPH), followed by lyophilization. The main parameters during antisolvent process including volume ratio of dimethyl sulfoxide (DMSO) and $\mathrm{H}_{2} \mathrm{O}$ and dripping speed were optimized and their effects on mean particle size (MPS) and yield of HCPT primary particles were investigated. In the high pressure homogeneous procedure, types of surfactants, amount of surfactants, and homogenization pressure (HP) were optimized and their influences on MPS, zeta potential (ZP), and morphology were analyzed. The optimum conditions of HCPTNPs were as follows: $0.2 \mathrm{mg} / \mathrm{mL} \mathrm{HCPT}$ aqueous suspension, $1 \%$ of ASS, 1000 bar of HP, and 20 passes. Finally, the HCPTNPs via lyophilization using glucose as lyoprotectant under optimum conditions had an MPS of $179.6 \mathrm{~nm}$ and a ZP of $28.79 \pm 1.97 \mathrm{mV}$. The short-term stability of HCPTNPs indicated that the MPS changed in a small range.
\end{abstract}

\section{Introduction}

10-Hydroxycamptothecin (HCPT), one analogue of camptothecin, has been shown to have a strong antitumor activity against a wide range of experimental tumors including gastric carcinoma, hepatoma, leukemia, and head and neck tumors [1]. Moreover, it has been proven to be more potent and less toxic in experimental animals and in human clinical evaluation compared to CPT. However, its poor solubility in water and in other physiologically acceptable organic solvents hinders its clinical application [2-6]. To improve the solubility and stability of HCPT, many new drug delivery systems including microspheres, liposomes, and micelles system were developed [7].
Nanoparticles have been successfully used to solve the poor solubility of HCPT $[6,8-14]$. Up to now, there are mainly two kinds of method [15]. One is to nucleate and assemble drug molecular into nanoparticles, which consists of two major approaches, preparation of micropowder [16] and surfactants [17]. Another is to prepare large drug particles into nanoparticles using high pressure homogenization [18]. In addition, supercritical fluid antisolvent (SAS) has also been widely studied $[19,20]$.

Though the nanoparticles prepared by SAS have a small and uniform particle size, defects of small batch and high production cost limit its practical use. However, the particles prepared by antisolvent precipitation method at atmospheric pressure have a wide particle size distribution. It is rapid 
and easy to operate and industrialize compared to SAS. In particular, it is suitable to prepare primary particles. High pressure homogenization ( $\mathrm{HPH})$ is an effective tool to prepare refined particles and emulsion products. The $\mathrm{HPH}$ technique has merits of reducing particle size of the preparation and obtaining a narrow and uniform particle size distribution over the other methods such as ultrasonication, milling, and high speed shear.

In our study, antisolvent precipitation (AP) at atmospheric pressure combined HPH was utilized to prepare HCPTNPs. The AP process parameters including volume ratio of dimethyl sulfoxide (DMSO) and $\mathrm{H}_{2} \mathrm{O}$ and dripping speed were optimized and their effects on mean particle size (MPS) and yield of HCPT were discussed. HCPT primary particles obtained under optimum conditions were further prepared into HCPTNPs using $\mathrm{HPH}$. The optimum $\mathrm{HPH}$ procedure including types of surfactants, amount of surfactants, and homogenization pressure (HP) was determined. Finally, the MPS, zeta potential (ZP), and short-term stability of HCPTNPs obtained under optimum HPH conditions via lyophilization were researched.

\section{Materials and Methods}

2.1. Materials. HCPT (>98\%) was provided by Hisun Pharmaceutical (Taizhou, China). Dimethyl sulfoxide (DMSO), phosphatidylcholine, sodium tauroglycocholate, poloxamer 188 , sodium sulfate, gelatin, and glucose were obtained from Sigma-Aldrich (St. Louis, MO, USA).

\subsection{Preparation of HCPT Primary Particles. The HCPT} primary particles were prepared by antisolvent precipitation. Firstly, $5 \mathrm{mg} / \mathrm{mL}$ of raw HCPT was dissolved in DMSO. Then the drug solution was totally injected into aqueous solution with different volume at different flow rates by injection pump (JZB-1800D, Chien-Yuan Medical Technology Co., Ltd., Changsha, China). The aqueous solution was being stirred for $10 \mathrm{~min}$ at a certain speed until the drug solution dripped off. Then HCPT suspension was centrifuged at 10,000 rpm for 15 minutes and washed for three times to eliminate organic solvent DMSO by water. The final precipitate was just HCPT nanoparticles. Each experiment was repeated three times.

2.3. Preparation of HCPTNPs. Fifty milliliters of $0.2 \mathrm{mg} / \mathrm{mL}$ HCPT primary particles aqueous suspension that added certain quality of surfactants was processed using HPH. Then HCPTNPs were obtained under the conditions of a certain $\mathrm{HP}$ and passes.

2.4. Optimization of HCPTNPs. Due to the hydrophilicity of surfactants, the solubility of HCPT can be enhanced to some degree through the combination of different kinds of surfactants. Therefore, six kinds of surfactants were chosen to be selected, namely, lecithin, sodium taurocholate, poloxamer 188 , anhydrous sodium sulfate (ASS), sodium dodecyl sulfate (SDS), and gelatin. The adding ratio of different kinds of surfactants, HP, and passes were optimized to obtain the optimum preparation conditions of HCPTNPs. The adding ratios of surfactants were according to volume ratio of preparation system. The volume ratios were separately $1 \%$ $(0.5 \mathrm{mg})$ and $5 \%(2.5 \mathrm{mg})$. The experiment design was shown in Table 1.

2.5. Particle Size and ZP Analysis. Dynamic light scattering (DLS) (ZetaPALS, Brookhaven Instruments) with a $\mathrm{He}-\mathrm{Ne}$ laser $(632.8 \mathrm{~nm}, 35 \mathrm{~mW})$ as light source was employed to detect MPS and ZP of samples. The sample was prepared by dilution using pure water and every measurement was repeated three times.

2.6. Morphology Observations. The morphology of particles was ascertained by SEM (Quanta 200, FEI). Samples were installed on aluminum stubs by means of doublesided carbon tape and sputter-coated with gold under argon atmosphere.

2.7. Lyophilization. Based on the optimization $\mathrm{HPH}$, the HCPTNPs aqueous suspension was added to glucose which was three times the amount of HCPTNPs. After mixing process, samples were continuously taken out and joined in $8 \mathrm{~mL}$ glass vials. The HCPTNPs were lyophilized by a Gamma 2-20 apparatus (Christ, Germany) to obtain dry powder with good physicochemical stability. Freezing temperature was set $-40^{\circ} \mathrm{C}$ for $2 \mathrm{~h}$ to process the samples. Subsequently, lyophilization was underway at $-40^{\circ} \mathrm{C}$ for $48 \mathrm{~h}$, followed by a secondary drying phase of $12 \mathrm{~h}$ at $20^{\circ} \mathrm{C}$.

2.8. Short-Term Stability in Purified Water of HCPTNPs. The redispersibility study of HCPTNPs via lyophilization in purified water was detected by DLS equipment at $36.7^{\circ} \mathrm{C}$. The MPS and ZP of freeze-dried HCPTNPs were analyzed at predetermined time intervals $(0,1,2,4,6$, and $8 \mathrm{~h})$. Moreover, HCPT primary particles, HCPTNPs, and freezedried HCPTNPs which had the same concentration of HCPT were separately dispersed in a single vial with $4 \mathrm{~mL}$ purified water. The dispersion appearances of three samples were captured by camera.

\section{Results and Discussion}

3.1. Experiment Analysis of HCPT Primary Particles. There are many factors that affect the preparation of HCPT using antisolvent method. In this experiment, volume ratio of DMSO and $\mathrm{H}_{2} \mathrm{O}$ and dripping speed were optimized through orthogonal experiments. The particle size along with yield of HCPT primary particles were chosen to be the response values. As shown in Table 2, the MPS and yield of HCPT primary particles changed irregularly with volume ratio of HCPT-DMSO and $\mathrm{H}_{2} \mathrm{O}$ and dripping speed. MPS in most of trials were larger than $1 \mu \mathrm{m}$. The yield of HCPT primary particles also plays an important role in the process cost. The yield and MPS were synthetically considered to obtain the optimized conditions, namely, $5 \mathrm{mg} / \mathrm{mL}$ of HCPT concentration, $1: 1$ of the volume ratio of DMSO and $\mathrm{H}_{2} \mathrm{O}$, $4 \mathrm{~mL} / \mathrm{min}$ of dripping speed, and $10 \mathrm{~min}$ of stirring time at $500 \mathrm{rpm}$. The obtained suspension was centrifuged at 
TABLE 1: Experimental design of preparation of HCPTNPs.

\begin{tabular}{lcccccc}
\hline HP & Lecithin & Sodium taurocholate & ASS & Poloxamer 188 & Gelatin & SDS \\
\hline \multirow{2}{*}{$600 \mathrm{bar}$} & $0.5 \mathrm{mg}$ & $0.5 \mathrm{mg}$ & $0.5 \mathrm{mg}$ & $0.5 \mathrm{mg}$ & $0.5 \mathrm{mg}$ & $0.5 \mathrm{mg}$ \\
& $2.5 \mathrm{mg}$ & $2.5 \mathrm{mg}$ & $2.5 \mathrm{mg}$ & $2.5 \mathrm{mg}$ & $2.5 \mathrm{mg}$ & $2.5 \mathrm{mg}$ \\
$800 \mathrm{bar}$ & $0.5 \mathrm{mg}$ & $0.5 \mathrm{mg}$ & $0.5 \mathrm{mg}$ & $0.5 \mathrm{mg}$ & $0.5 \mathrm{mg}$ & $0.5 \mathrm{mg}$ \\
& $2.5 \mathrm{mg}$ & $2.5 \mathrm{mg}$ & $2.5 \mathrm{mg}$ & $2.5 \mathrm{mg}$ & $2.5 \mathrm{mg}$ & $2.5 \mathrm{mg}$ \\
$1000 \mathrm{bar}$ & $0.5 \mathrm{mg}$ & $0.5 \mathrm{mg}$ & $0.5 \mathrm{mg}$ & $0.5 \mathrm{mg}$ & $0.5 \mathrm{mg}$ & $0.5 \mathrm{mg}$ \\
& $2.5 \mathrm{mg}$ & $2.5 \mathrm{mg}$ & $2.5 \mathrm{mg}$ & $2.5 \mathrm{mg}$ & $2.5 \mathrm{mg}$ & $2.5 \mathrm{mg}$ \\
\hline
\end{tabular}

TABLE 2: The factors of orthogonal and experimental result.

\begin{tabular}{|c|c|c|c|}
\hline $\begin{array}{l}\text { Volume ratio of } \\
\text { DMSO and } \mathrm{H}_{2} \mathrm{O}\end{array}$ & $\begin{array}{c}\text { Dripping speed } \\
(\mathrm{mL} / \mathrm{min})\end{array}$ & MPS (nm) & Yield (\%) \\
\hline \multirow{5}{*}{$1: 1$} & 1 & 1284.8 & 82.8 \\
\hline & 2 & 991.6 & 82.6 \\
\hline & 3 & 1123.4 & 80.8 \\
\hline & 4 & 896.4 & 84.6 \\
\hline & 5 & 1077 & 83.0 \\
\hline \multirow{5}{*}{$1: 3$} & 1 & 1243.1 & 72.2 \\
\hline & 2 & 1005.6 & 77.2 \\
\hline & 3 & 1082.8 & 74.4 \\
\hline & 4 & 1044.1 & 77.0 \\
\hline & 5 & 1175.2 & 73.0 \\
\hline \multirow{5}{*}{$1: 5$} & 1 & 1275.7 & 72.4 \\
\hline & 2 & 820.6 & 76.6 \\
\hline & 3 & 961.9 & 73.8 \\
\hline & 4 & 1079.5 & 76.2 \\
\hline & 5 & 1011.9 & 71.2 \\
\hline
\end{tabular}

10,000 rpm for $15 \mathrm{~min}$. According to the optimum conditions of antisolvent method, ultrasonication was utilized to accomplish the antisolvent method. When dripping was finished, ultrasonication was going on for $10 \mathrm{~min}$. The MPS and ZP of HCPT primary particles were $746 \mathrm{~nm}$ and $-23.73 \mathrm{mV}$, separately. The particle size of drugs has an important effect in the distribution in vivo. The HCPT with large particle size has low water solubility, resulting in difficulty to shuttle across cell membranes and playing a pharmacodynamic role. So the particle size has the key role, depending on the distribution and utilization in vivo.

3.2. Surface Morphology of HCPT Primary Particles. The morphology of raw HCPT and HCPT primary particles was observed by scanning electron microscopy (SEM). In Figure 1(a), raw HCPT was in irregular lump crystal form with a width of $1-30 \mu \mathrm{m}$ range. However, HCPT primary particles showed sphere morphology and uniform size. Moreover, there were not visible drug crystals.

\subsection{Optimization of HCPTNPs Using $\mathrm{HPH}$}

3.3.1. Effects of Surfactants on MPS and ZP of HCPTNPs. Firstly, $0.2 \mathrm{mg} / \mathrm{mL}$ HCPT aqueous suspension was added to lecithin according to the $5 \%$ of mass ratio of $\mathrm{HCPT}$ powder and $5 \%$ of volume ratio of preparation system, respectively. Ultrasonication was used to make lecithin totally dissolve. HP of 600 bar, 800 bar, and 1000 bar were chosen in the homogenization process. Twenty passes were conducted under each HP. The MPS and ZP of HCPTNPs were detected under three different pressures. The results were shown in Table 3. Seen from Table 3, the MPS of HCPTNPs increased with the increasing of lecithin addition. The ZP of HCPTNPs also varied with the increasing of lecithin addition. Seen from Figure 2, homogeneous degree between HCPT and lecithin was improved with the increase of $\mathrm{HPH}$ and passes, which caused that the MPS of HCPTNPs with $0.5 \mathrm{mg}$ lecithin maintained 230-240 nm. Meanwhile, the MPS of HCPTNPs with $2.5 \mathrm{mg}$ lecithin maintained $260-270 \mathrm{~nm}$. The MPS of HCPTNPs was smaller, when addition of lecithin was $5 \%$ of mass ratio of HCPT powder and HP was 1000 bar. However, seen from SEM images, HCPTNPs with lecithin unevenly distributed and presented adhesion phenomenon in irregular shape.

The preparation of HCPTNPs using sodium taurocholate as surfactants was referred to Section 2.3. The results were shown in Table 4. From Figure 3, MPS got larger when the sodium taurocholate was added more under the same HP 


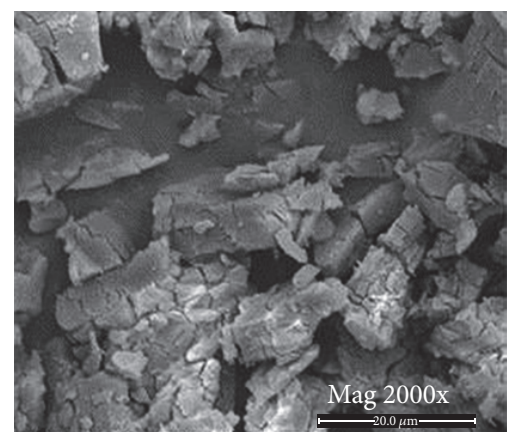

(a)

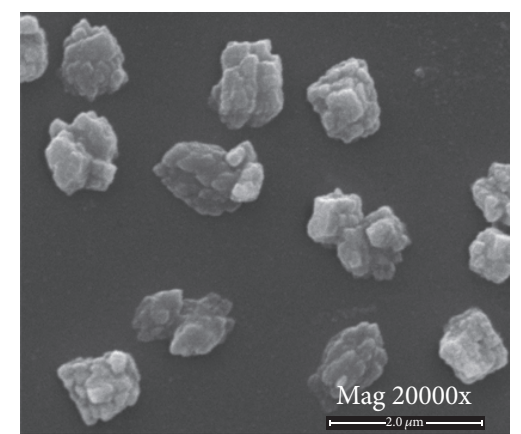

(b)

FIGURE 1: SEM images of (a) raw HCPT and (b) HCPT primary particles.

TABle 3: Preparation of HCPTNPs with lecithin by HPH.

\begin{tabular}{lccc}
\hline Pressure (bar) & Mass $(\mathrm{mg})$ & MPS $(\mathrm{nm})$ & ZP $(\mathrm{mV})$ \\
\hline \multirow{2}{*}{600} & 0.5 & 85.2 & $11.91 \pm 0.92$ \\
& 2.5 & 143.1 & $-24.00 \pm 1.27$ \\
800 & 0.5 & 233.6 & $-31.35 \pm 0.44$ \\
& 2.5 & 272.2 & $-41.73 \pm 1.94$ \\
1000 & 0.5 & 258.3 & $-33.23 \pm 2.01$ \\
& 2.5 & 261.7 & $-27.32 \pm 1.43$ \\
\hline
\end{tabular}

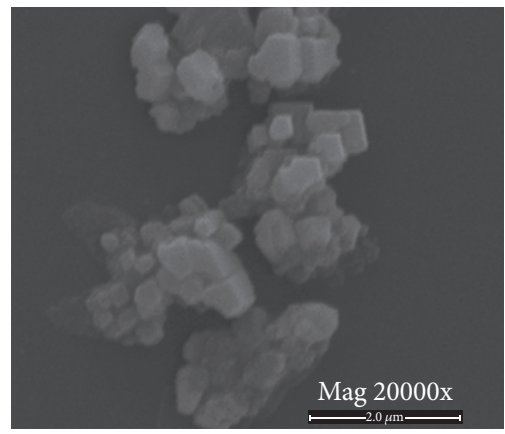

(a)

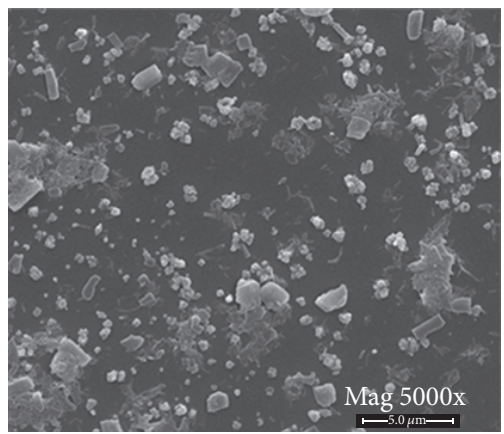

(d)

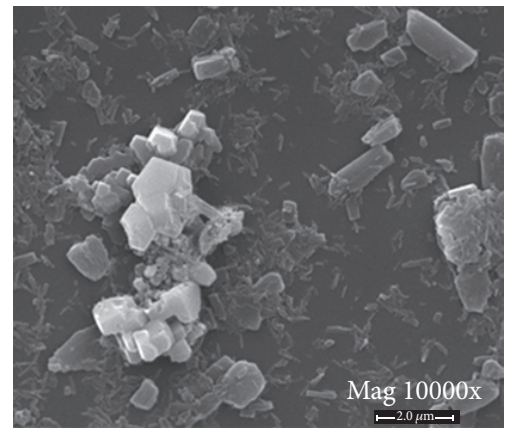

(b)

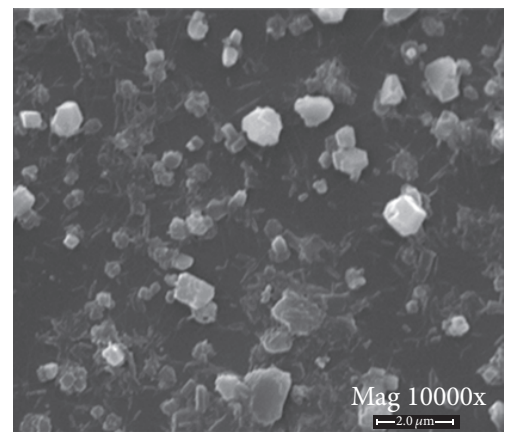

(e)

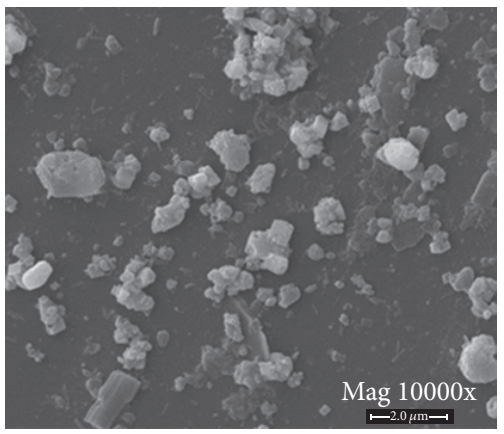

(c)

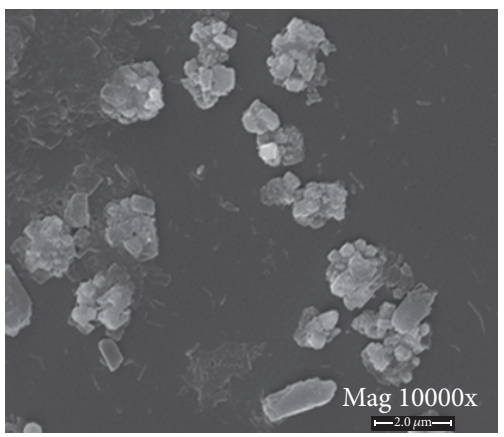

(f)

FIGURE 2: SEM images of HCPTNPs using lecithin as surfactants under different HP and amount conditions: (a) $0.5 \mathrm{mg}, 600 \mathrm{bar}$; (b) $0.5 \mathrm{mg}$, 800 bar; (c) $0.5 \mathrm{mg}, 1000$ bar; (d) $2.5 \mathrm{mg}, 600$ bar; (e) $2.5 \mathrm{mg}, 800$ bar; (f) $2.5 \mathrm{mg}, 1000$ bar. 
TABLE 4: Preparation of HCPTNPs with sodium taurocholate by HPH.

\begin{tabular}{lccr}
\hline Pressure (bar) & Mass $(\mathrm{mg})$ & MPS $(\mathrm{nm})$ & ZP $(\mathrm{mV})$ \\
\hline \multirow{2}{*}{600} & 0.5 & 269.0 & $-23.12 \pm 1.05$ \\
& 2.5 & 329.9 & $-26.22 \pm 1.08$ \\
800 & 0.5 & 333.6 & $-31.92 \pm 0.18$ \\
& 2.5 & 398.6 & $-22.37 \pm 1.44$ \\
1000 & 0.5 & 341.8 & $-25.06 \pm 0.98$ \\
& 2.5 & 289.2 & $-30.9 \pm 0.53$ \\
\hline
\end{tabular}

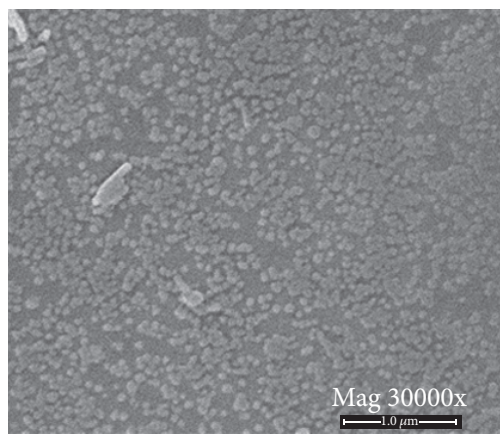

(a)

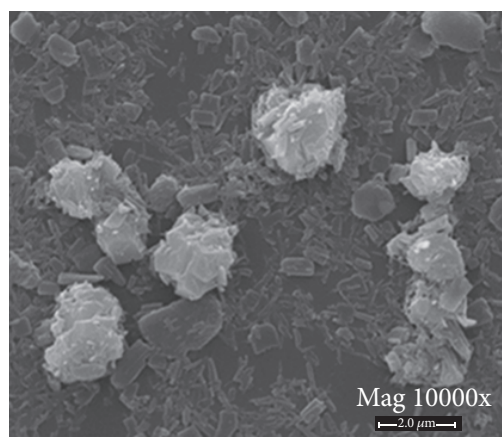

(d)

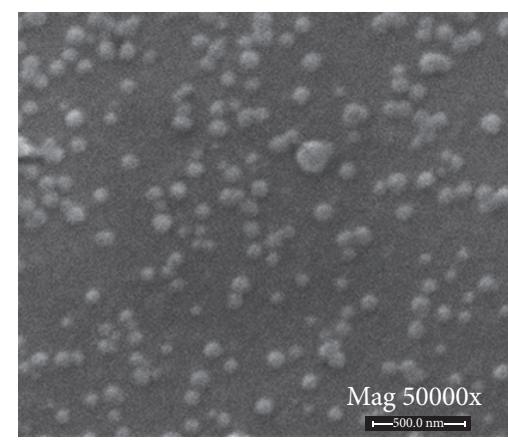

(b)

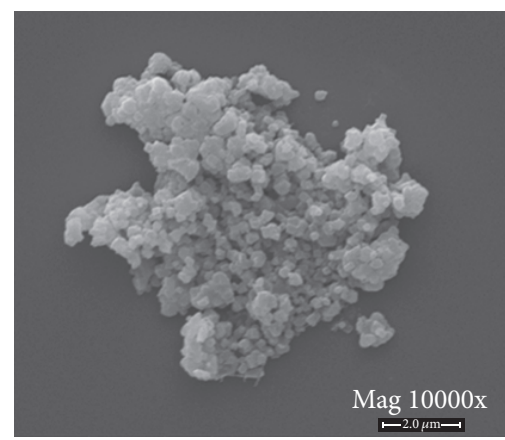

(e)

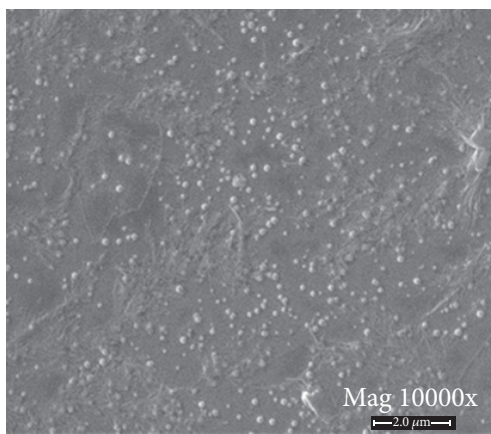

(c)

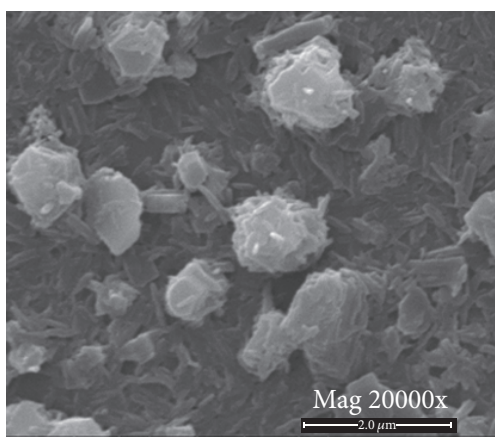

(f)

FIGURE 3: SEM images of HCPTNPs using sodium taurocholate as surfactants under different HP and amount conditions: (a) $0.5 \mathrm{mg}$, 600 bar; (b) $0.5 \mathrm{mg}, 800 \mathrm{bar}$; (c) $0.5 \mathrm{mg}, 1000 \mathrm{bar}$; (d) $2.5 \mathrm{mg}, 600 \mathrm{bar}$; (e) $2.5 \mathrm{mg}, 800 \mathrm{bar}$ (f) $2.5 \mathrm{mg}$, $1000 \mathrm{bar}$.

condition. So the amount of surfactants had an effect on MPS of HCPTNPs. When the addition of sodium taurocholate was $0.5 \mathrm{mg}$, MPS of HCPTNPs got larger and was distributed in $270 \mathrm{~nm}-340 \mathrm{~nm}$ with the increase of HP. Meanwhile, when the addition of sodium taurocholate was $2.5 \mathrm{mg}$, MPS of HCPTNPs was about $300 \mathrm{~nm}$ with the increase of HP. Seen from SEM images, the HCPTNPs dispersed well, had a smooth surface, and had a large particle size. Adhesion happened to HCPTNPs with the amount rising of sodium taurocholate.

The experiment result using poloxamer 188 as surfactants was shown in Table 5 . As seen in Table 5, the MPS of HCPTNPs decreased and varied within $150-270 \mathrm{~nm}$ with the increase of HP when $0.5 \mathrm{mg}$ poloxamer 188 was added. However, the MPS of HCPTNPs had a tendency to become large when $2.5 \mathrm{mg}$ poloxamer 188 was added. So the amount
TABLE 5: Preparation of HCPTNPs with poloxamer 188 by HPH.

\begin{tabular}{lccc}
\hline Pressure (bar) & Mass $(\mathrm{mg})$ & MPS $(\mathrm{nm})$ & ZP $(\mathrm{mV})$ \\
\hline \multirow{2}{*}{600} & 0.5 & 272.6 & $-21.02 \pm 1.82$ \\
& 2.5 & 181.9 & $-28.11 \pm 1.25$ \\
800 & 0.5 & 214.1 & $-27.42 \pm 1.53$ \\
& 2.5 & 147.3 & $-14.79 \pm 1.36$ \\
1000 & 0.5 & 144.3 & $-18.57 \pm 1.33$ \\
& 2.5 & 273.6 & $-26.22 \pm 1.90$ \\
\hline
\end{tabular}

of surfactant could make an influence on MPS of HCPT nanocolloidal particles. The amount of poloxamer 188 was added more; the MPS of HCPTNPs was larger. Seen from Figure 4, HCPTNPs have defects of conglutination, nonuniform particle size distribution. 


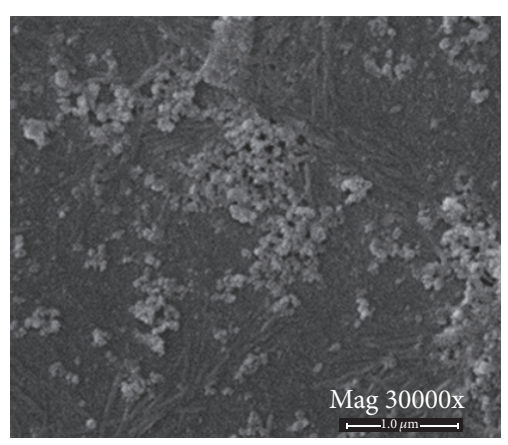

(a)

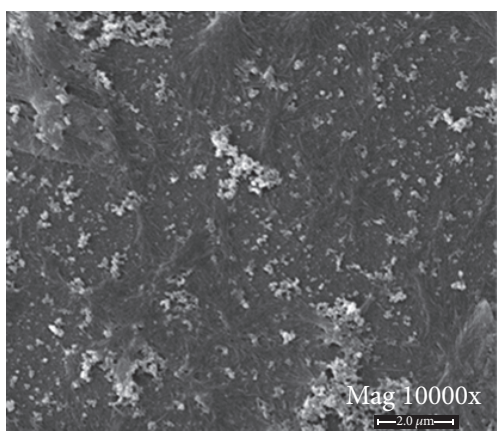

(d)

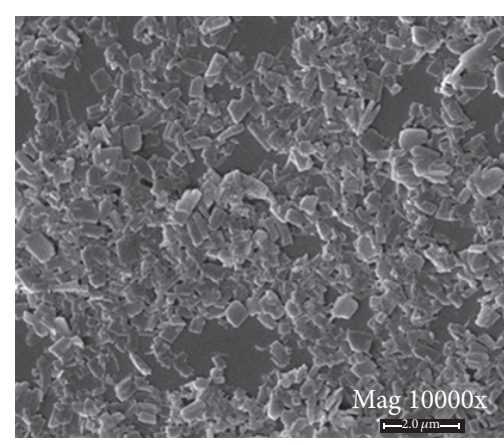

(b)

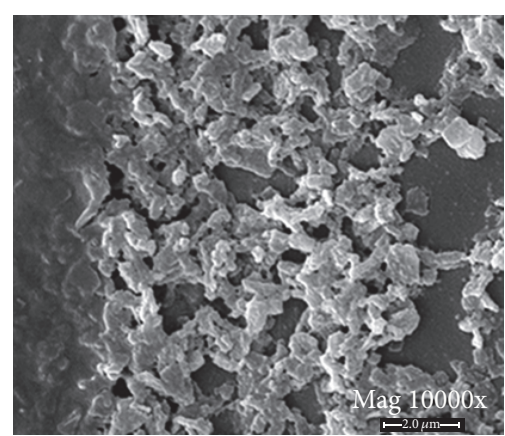

(e)

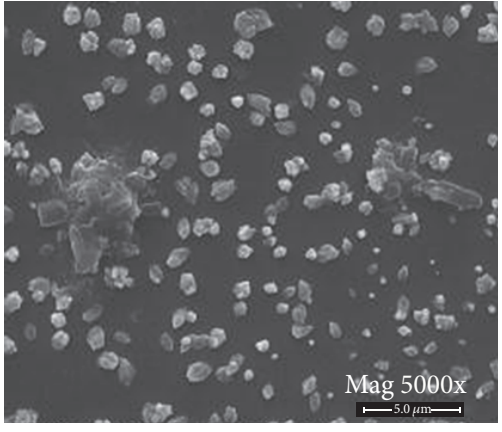

(c)

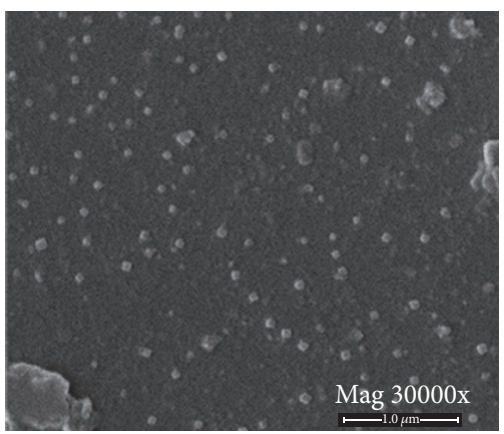

(f)

FIGURE 4: SEM images of HCPTNPs using poloxamer 188 as surfactants under different HP and amount conditions: (a) 0.5 mg, 600 bar; (b) $0.5 \mathrm{mg}, 800 \mathrm{bar}$; (c) $0.5 \mathrm{mg}, 1000 \mathrm{bar}$ (d) $2.5 \mathrm{mg}$, $600 \mathrm{bar}$; (e) $2.5 \mathrm{mg}$, $800 \mathrm{bar}$; (f) $2.5 \mathrm{mg}, 1000$ bar.

TABLE 6: Preparation of HCPTNPs with ASS by HPH.

\begin{tabular}{lccr}
\hline Pressure (bar) & Mass $(\mathrm{mg})$ & MPS $(\mathrm{nm})$ & ZP $(\mathrm{mV})$ \\
\hline \multirow{2}{*}{600} & 0.5 & 229.5 & $-32.44 \pm 1.47$ \\
& 2.5 & 147.5 & $-34.39 \pm 1.18$ \\
800 & 0.5 & 154.9 & $-37.57 \pm 0.98$ \\
& 2.5 & 224.6 & $-25.03 \pm 1.86$ \\
1000 & 0.5 & 168.7 & $-35.14 \pm 0.69$ \\
& 2.5 & 250.6 & $-26.58 \pm 1.73$ \\
\hline
\end{tabular}

As seen in Table 6, the MPS of HCPTNPs increased with the increasing of lecithin addition. The ZP of HCPTNPs also varied with the increasing of lecithin addition. The MPS of HCPTNPs decreased and varied within $236-168 \mathrm{~nm}$ with the increase of HP when $0.5 \mathrm{mg}$ ASS was added. However, the MPS of HCPTNPs had an inapparent tendency to become small and varied within 450-234 $\mathrm{nm}$ when $2.5 \mathrm{mg}$ ASS was added. Figure 5 indicated that HCPTNPs had a uniform particle size distribution, no visible particles, and spherical and smooth particles.

Table 7 indicated the amount of SDS was added more; the MPS of HCPTNPs was larger. The MPS of HCPTNPs decreased and varied within $230-169 \mathrm{~nm}$ with the increase of HP and passes when $0.5 \mathrm{mg}$ SDS was added. Compared with $0.5 \mathrm{mg}$ SDS, the MPS of HCPTNPs added to $2.5 \mathrm{mg}$ SDS was larger varying within $150-250 \mathrm{~nm}$. Figure 6 showed that
HCPTNPs had a nonuniform particle size distribution and phenomenon of agglomeration.

As shown in Table 8, when the amount of gelatin was kept the same, HP was larger and passes were more, and the MPS of HCPTNPs was smaller. The MPS of HCPTNPs added to $2.5 \mathrm{mg}$ gelatin was smaller in comparison with the MPS of HCPTNPs added to $0.5 \mathrm{mg}$ gelatin. The MPS varied within $190-75 \mathrm{~nm}$. The SEM images of Figure 7 indicated that HCPTNPs using gelatin as surfactants showed adhesion and had an uneven particle size distribution.

3.3.2. Optimum HPH Conditions of HCPTNPs. MPS, ZP, HP, adding amount of surfactants, and morphology of HCPTNPs were all taken into account; ASS was chosen to be the best surfactant. The optimum conditions were as follows: $0.2 \mathrm{mg} / \mathrm{mL}$ HCPT aqueous suspension, $1 \%$ of ASS, $1000 \mathrm{bar}$ 


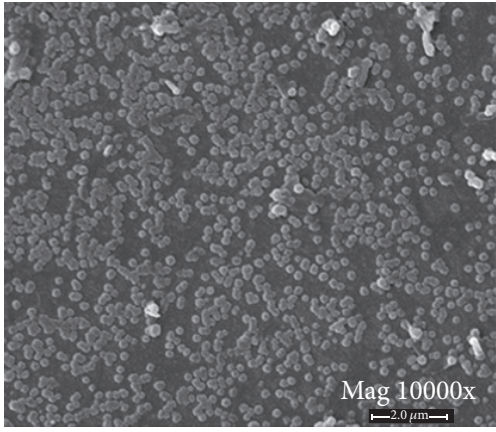

(a)

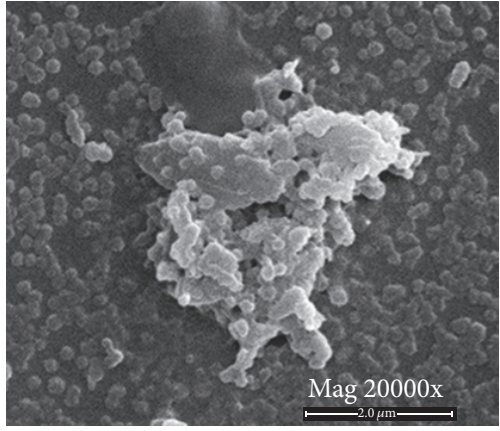

(d)

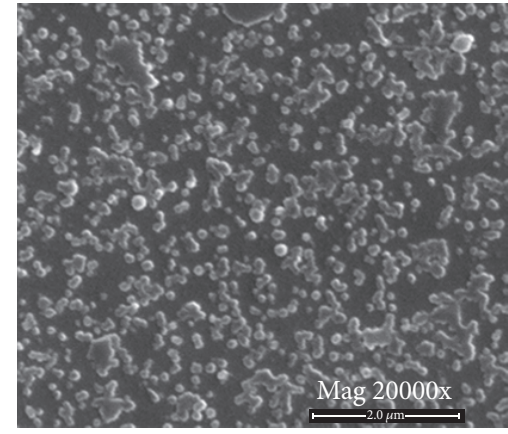

(b)

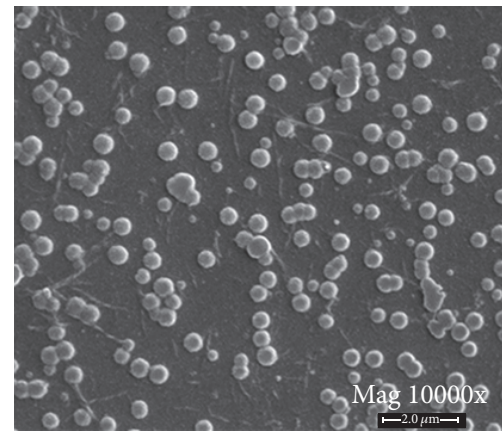

(e)

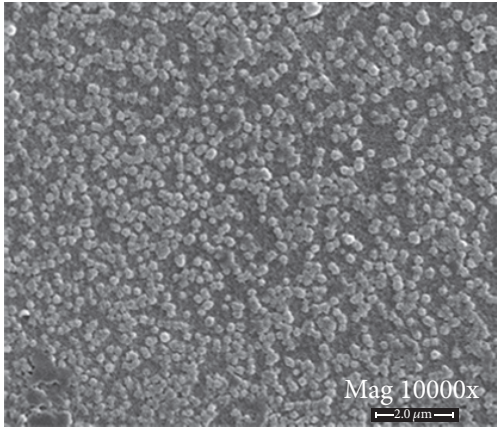

(c)

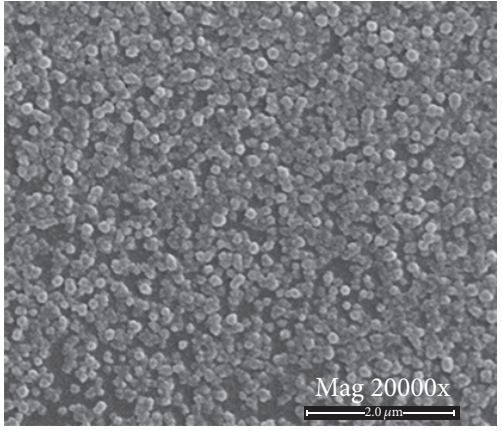

(f)

FIGURE 5: SEM images of HCPTNPs using ASS as surfactants under different HP and amount conditions: (a) $0.5 \mathrm{mg}, 600 \mathrm{bar}$; (b) $0.5 \mathrm{mg}$, 800 bar; (c) $0.5 \mathrm{mg}$, 1000 bar; (d) $2.5 \mathrm{mg}, 600$ bar; (e) $2.5 \mathrm{mg}$, 800 bar; (f) $2.5 \mathrm{mg}, 1000$ bar.

TABLE 7: Preparation of HCPTNPs with SDS by HPH.

\begin{tabular}{lccr}
\hline Pressure (bar) & Mass $(\mathrm{mg})$ & MPS $(\mathrm{nm})$ & ZP $(\mathrm{mV})$ \\
\hline \multirow{2}{*}{600} & 0.5 & 229.5 & $-32.44 \pm 1.47$ \\
& 2.5 & 147.5 & $-34.39 \pm 1.18$ \\
800 & 0.5 & 154.9 & $-37.57 \pm 0.98$ \\
& 2.5 & 224.6 & $-25.03 \pm 1.86$ \\
1000 & 0.5 & 168.7 & $-35.14 \pm 0.69$ \\
& 2.5 & 250.6 & $-26.58 \pm 1.73$ \\
\hline
\end{tabular}

TABLE 8: Preparation of HCPTNPs with gelatin by HPH.

\begin{tabular}{lccr}
\hline Pressure (bar) & Mass $(\mathrm{mg})$ & MPS $(\mathrm{nm})$ & ZP $(\mathrm{mV})$ \\
\hline \multirow{2}{*}{600} & 0.5 & 433.5 & $-22.51 \pm 1.16$ \\
& 2.5 & 191.5 & $-12.42 \pm 0.49$ \\
800 & 0.5 & 153.8 & $-32.35 \pm 0.56$ \\
& 2.5 & 169.1 & $-36.46 \pm 1.25$ \\
1000 & 0.5 & 163.3 & $-31.55 \pm 1.16$ \\
& 2.5 & 74.9 & $-23.04 \pm 1.04$ \\
\hline
\end{tabular}

of HP, and 20 passes. Under the optimum conditions, the MPS and ZP of HCPTNPs were $168.6 \mathrm{~nm}$ and $-25.18 \mathrm{mV}$, respectively.

3.4. Short-Term Stability in Purified Water of Freeze-Dried HCPTNPs. As seen in Table 9, MPS and ZP of freezedried HCPTNPs had minor change, which showed that freeze-dried HCPTNPs were physically and chemically stable for up to $8 \mathrm{~h}$ at room temperature. The precept of shortterm stability study was derived from the evaluation of internationally marketable Abraxane from FDA (US Food and Drug Administration). Seen from Figure 8, the disperse states of HCPTNPs via HPH and freeze-dried HCPTNPs in water were both opalescent and showed no significant difference. Meanwhile, due to large particle size of HCPT primary particles, the water dispersant seemed turbid. In 


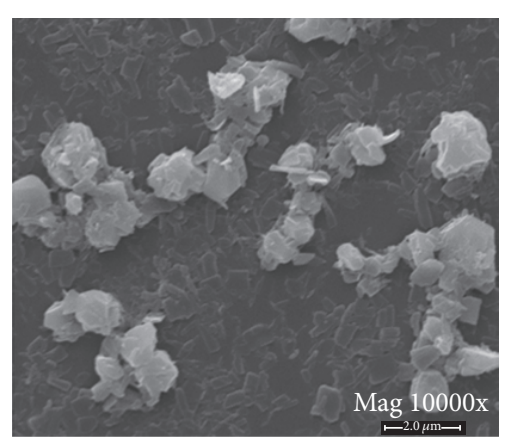

(a)

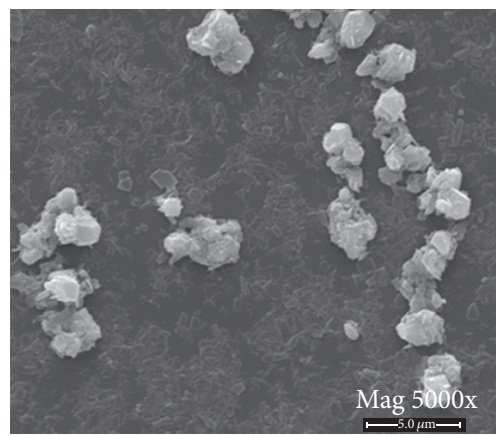

(d)

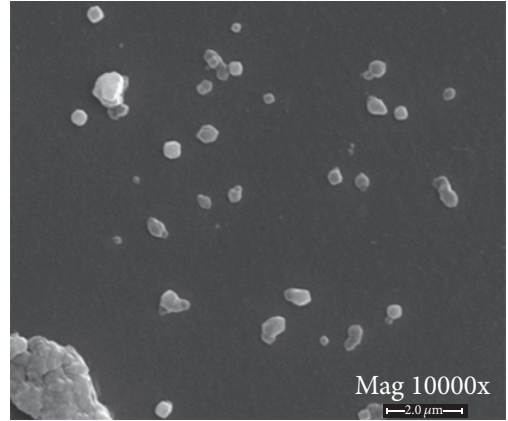

(b)

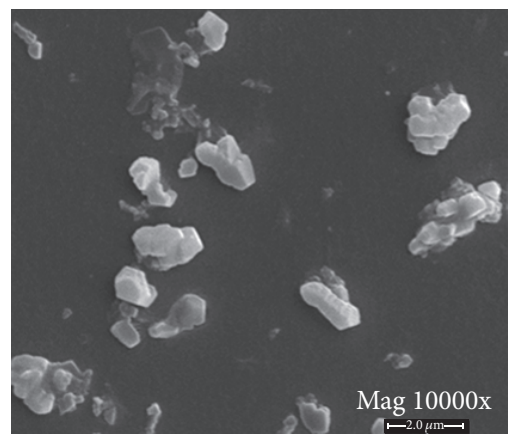

(e)

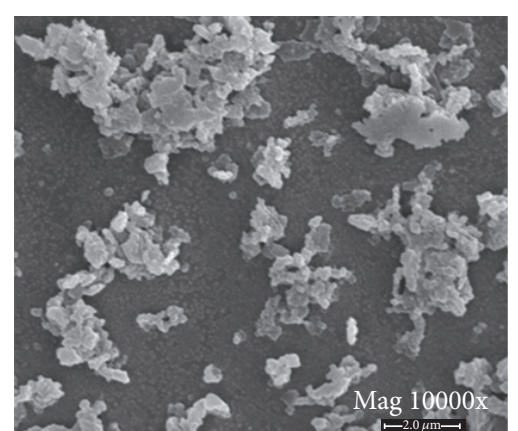

(c)

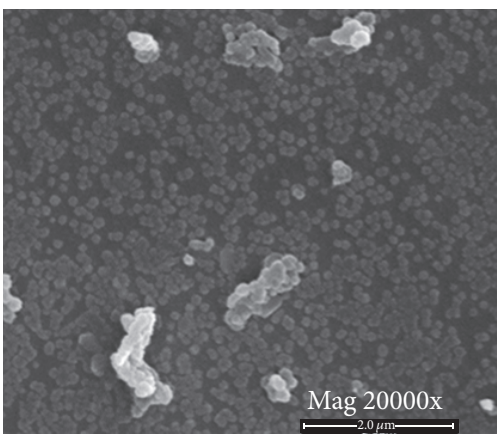

(f)

Figure 6: SEM images of HCPTNPs using SDS as surfactants under different HP and amount conditions: (a) $0.5 \mathrm{mg}, 600 \mathrm{bar}$; (b) $0.5 \mathrm{mg}$, 800 bar; (c) $0.5 \mathrm{mg}, 1000$ bar; (d) $2.5 \mathrm{mg}, 600$ bar; (e) $2.5 \mathrm{mg}$, 800 bar; (f) $2.5 \mathrm{mg}$, 1000 bar.

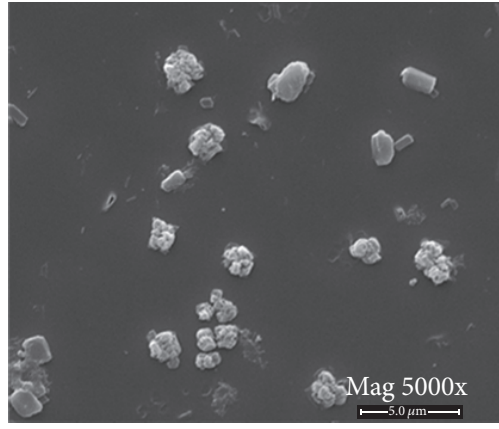

(a)

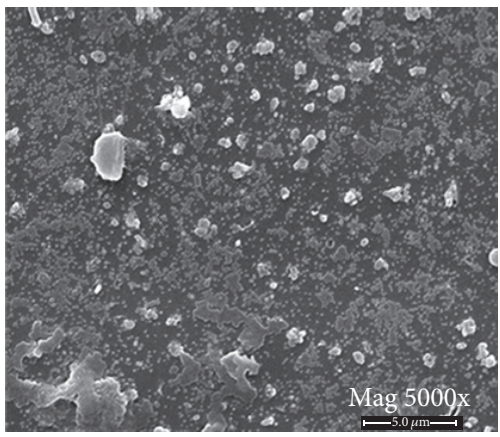

(d)

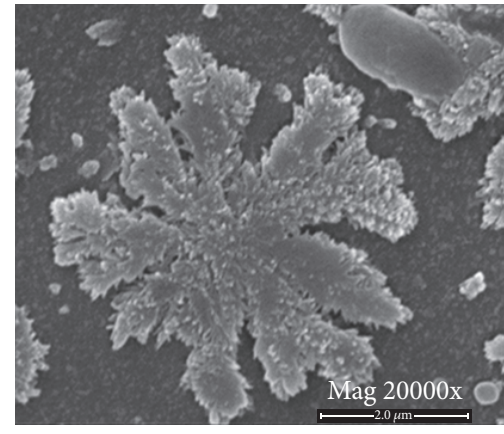

(b)

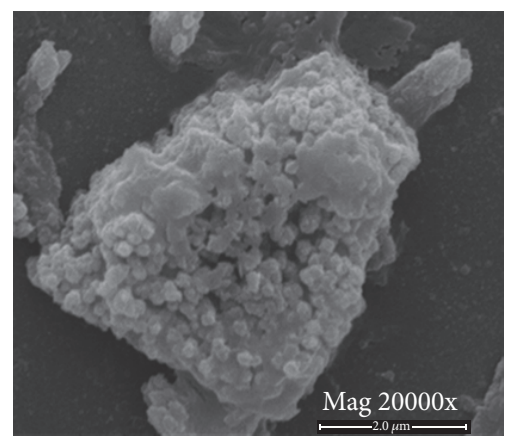

(e)

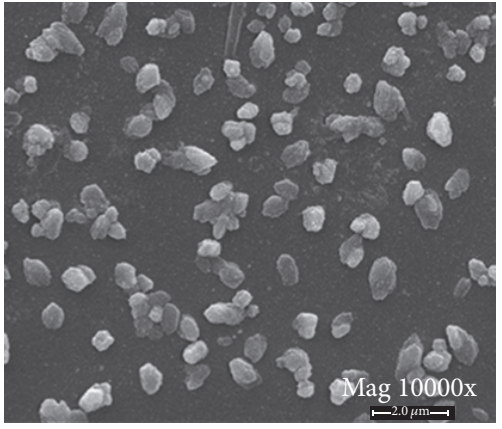

(c)

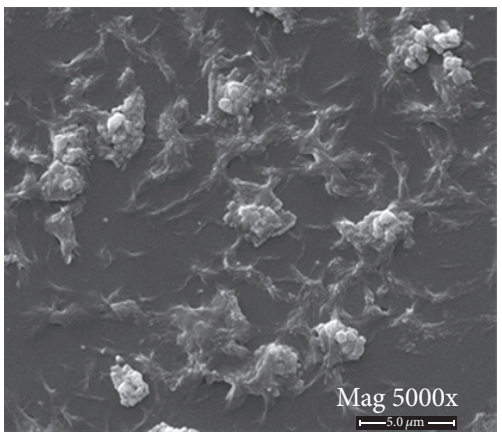

(f)

FIGURE 7: SEM images of HCPTNPs using gelatin as surfactants under different HP and amount conditions: (a) $0.5 \mathrm{mg}$, $600 \mathrm{bar}$; (b) $0.5 \mathrm{mg}$, 800 bar; (c) $0.5 \mathrm{mg}, 1000$ bar; (d) $2.5 \mathrm{mg}, 600$ bar; (e) $2.5 \mathrm{mg}, 800$ bar; (f) $2.5 \mathrm{mg}, 1000$ bar. 


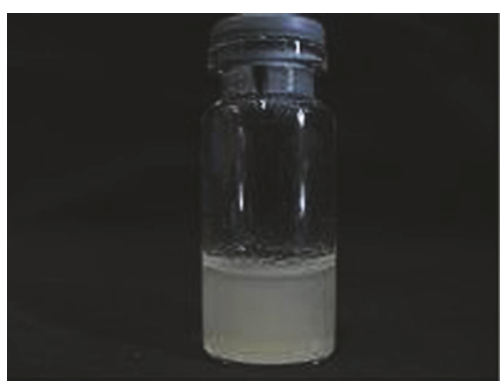

(a)

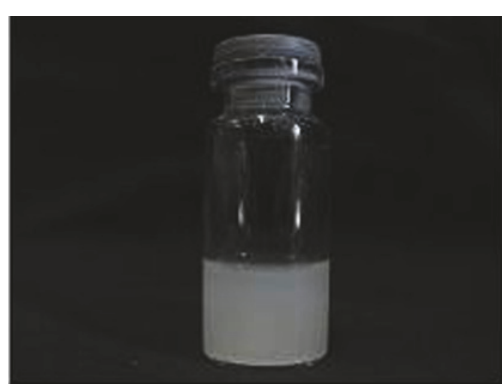

(b)

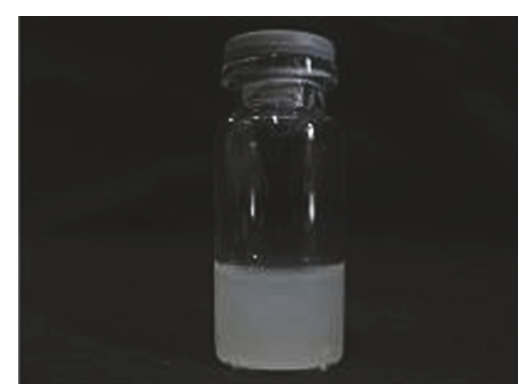

(c)

FIGURE 8: Dispersion appearances in water of (a) HCPT primary particles, (b) HCPT nanocolloidal particles, and (c) freeze-dried HCPT nanocolloidal particles.

TABLE 9: Redispersibility of freeze-dried HCPTNPs in purified water.

\begin{tabular}{lcc}
\hline Time $(\mathrm{h})$ & MPS $(\mathrm{nm})$ & $\mathrm{ZP}(\mathrm{mV})$ \\
\hline 0 & 179.6 & $-28.79 \pm 1.97$ \\
1 & 193.3 & $-27.99 \pm 2.21$ \\
2 & 221.3 & $-29.98 \pm 0.74$ \\
4 & 235.4 & $-23.70 \pm 2.08$ \\
6 & 247.1 & $-27.87 \pm 1.37$ \\
8 & 245.8 & $-30.02 \pm 0.67$ \\
\hline
\end{tabular}

particular, some drug particles appeared upon the surface of the water.

\section{Conclusions}

It had been shown that antisolvent method combined with HPH could be used to prepare HCPT nanocolloidal particles, which was physically and chemically stable in the short run. In this study, preparation technology of HCPTNPs was optimized. Characters of HCPTNPs were determined, which included MPS, ZP, redispersibility in purified water, and SEM. The results indicated that freeze-dried HCPTNPs had a uniform particle size and good stability in the short run, which demonstrated a promising prospect of the new formulation of HCPT. However, long-term stability study and in vivo research of HCPTNPs should now be proceeded to provide references for therapeutic use.

\section{Abbreviations}

AP: Antisolvent precipitation

ASS: Anhydrous sodium sulfate

DLS: Dynamic light scattering

DMSO: Dimethyl sulfoxide

HCPT: 10-Hydroxycamptothecin

HP: Homogenization pressure

$\mathrm{HPH}$ : High pressure homogenization

MPS: $\quad$ Mean particle size

SAS: $\quad$ Supercritical fluid antisolvent

SDS: Sodium dodecyl sulfate

SEM: Scanning electron microscope

ZP: Zeta potential.

\section{Conflicts of Interest}

There are no conflicts of interest to declare.

\section{Acknowledgments}

The authors would also like to acknowledge the financial support from the National Natural Science Foundation of China (no. 21473023).

\section{References}

[1] Y.-F. Li and R. Zhang, "Reversed-phase high-performance liquid chromatography method for the simultaneous quantitation of the lactone and carboxylate forms of the novel natural product anticancer agent 10-hydroxycamptothecin in biological fluids and tissues," Journal of Chromatography B: Biomedical Applications, vol. 686, no. 2, pp. 257-265, 1996.

[2] T. G. Burke and Z. Mi, "Preferential binding of the carboxylate form of camptothecin by human serum albumin," Analytical Biochemistry, vol. 212, no. 1, pp. 285-287, 1993.

[3] A. Hatefi and B. Amsden, "Camptothecin delivery methods," Pharmaceutical Research, vol. 19, no. 10, pp. 1389-1399, 2002.

[4] L. P. Rivory and J. Robert, "Reversed-phase high-performance liquid chromatographic method for the simultaneous quantitation of the carboxylate and lactone forms of the camptothecin derivative irinotecan, CPT-11, and its metabolite SN-38 in plasma," Journal of Chromatography B: Biomedical Sciences and Applications, vol. 661, no. 1, pp. 133-141, 1994.

[5] T. Tadros, P. Izquierdo, J. Esquena, and C. Solans, "Formation and stability of nano-emulsions," Advances in Colloid and Interface Science, vol. 108-109, pp. 303-318, 2004.

[6] L. Yang, F. Cui, D. Cun, A. Tao, K. Shi, and W. Lin, "Preparation, characterization and biodistribution of the lactone form of 10hydroxycamptothecin (HCPT)-loaded bovine serum albumin (BSA) nanoparticles," International Journal of Pharmaceutics, vol. 340, no. 1-2, pp. 163-172, 2007.

[7] R. Zhang, Y. Li, Q. Cai, T. Liu, H. Sun, and B. Chambless, "Preclinical pharmacology of the natural product anticancer agent 10-hydroxycamptothecin, an inhibitor of topoisomerase I," Cancer Chemotherapy and Pharmacology, vol. 41, no. 4, pp. 257-267, 1998.

[8] D. Chena, S. Chena, Q. Wanga, P. Guoa, Y. Caoa, and D. Lib, "Intracellular delivery of 10-hydroxycamptothecin with novel solid lipid nanoparticles (SLN) against multidrug resistance," 
Abstracts/Journal of Controlled Release, vol. 172, pp. e14-e97, 2013.

[9] M. Hong, S. Zhu, Y. Jiang et al., "Novel anti-tumor strategy: PEG-hydroxycamptothecin conjugate loaded transferrin-PEGnanoparticles," Journal of Controlled Release, vol. 141, no. 1, pp. 22-29, 2010.

[10] J.-B. Qu, H.-H. Shao, G.-L. Jing, and F. Huang, "PEG-chitosancoated iron oxide nanoparticles with high saturated magnetization as carriers of 10-hydroxycamptothecin: preparation, characterization and cytotoxicity studies," Colloids and Surfaces B: Biointerfaces, vol. 102, pp. 37-44, 2013.

[11] J. Wang, R. Wang, and L. B. Li, "Preparation and properties of hydroxycamptothecin-loaded nanoparticles made of amphiphilic copolymer and normal polymer," Journal of Colloid and Interface Science, vol. 336, no. 2, pp. 808-813, 2009.

[12] Z. Yang, W. Gong, Z. Wang et al., "A novel drugpolyethylene glycol liquid compound method to prepare 10-hydroxycamptothecin loaded human serum albumin nanoparticle," International Journal of Pharmaceutics, vol. 490, no. 1-2, pp. 412-428, 2015.

[13] L. Zhang, M. Yang, Q. Wang et al., "10-Hydroxycamptothecin loaded nanoparticles: preparation and antitumor activity in mice," Journal of Controlled Release, vol. 119, no. 2, pp. 153-162, 2007.

[14] X. Zhao, R. Jiang, Y. Zu et al., "Process optimization studies of 10-Hydroxycamptothecin (HCPT)-loaded folate-conjugated chitosan nanoparticles by SAS-ionic crosslink combination using response surface methodology (RSM)," Applied Surface Science, vol. 258, no. 6, pp. 2000-2005, 2012.

[15] B. van Eerdenbrugh, L. Froyen, J. A. Martens et al., "Characterization of physico-chemical properties and pharmaceutical performance of sucrose co-freeze-dried solid nanoparticulate powders of the anti-HIV agent loviride prepared by media milling," International Journal of Pharmaceutics, vol. 338, no. 12, pp. 198-206, 2007.

[16] T. L. Rogers, I. B. Gillespie, J. E. Hitt et al., "Development and characterization of a scalable controlled precipitation process to enhance the dissolution of poorly water-soluble drugs," Pharmaceutical Research, vol. 21, no. 11, pp. 2048-2057, 2004.

[17] P. Kocbek, S. Baumgartner, and J. Kristl, "Preparation and evaluation of nanosuspensions for enhancing the dissolution of poorly soluble drugs," International Journal of Pharmaceutics, vol. 312, no. 1-2, pp. 179-186, 2006.

[18] K. P. Krause and R. H. Müller, "Production and characterisation of highly concentrated nanosuspensions by high pressure homogenisation," International Journal of Pharmaceutics, vol. 214, no. 1-2, pp. 21-24, 2001.

[19] E. Reverchon, G. Della Porta, and P. Pallado, "Supercritical antisolvent precipitation of salbutamol microparticles," Powder Technology, vol. 114, no. 1-3, pp. 17-22, 2001.

[20] X. Zhao, Y. Zu, R. Jiang et al., "Preparation and physicochemical properties of 10-hydroxycamptothecin (HCPT) nanoparticles by supercritical antisolvent (SAS) process," International Journal of Molecular Sciences, vol. 12, no. 4, pp. 2678-2691, 2011. 

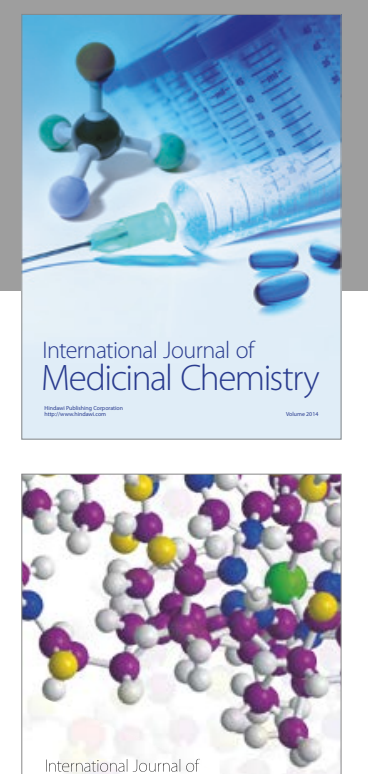

Carbohydrate Chemistry

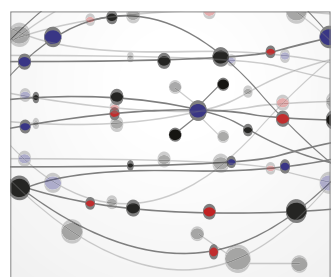

The Scientific World Journal
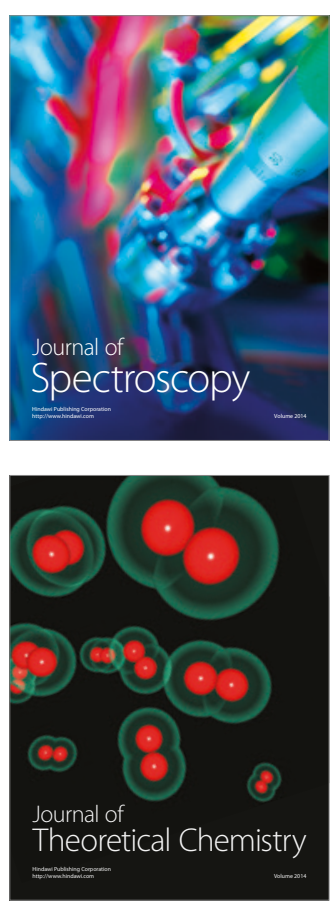
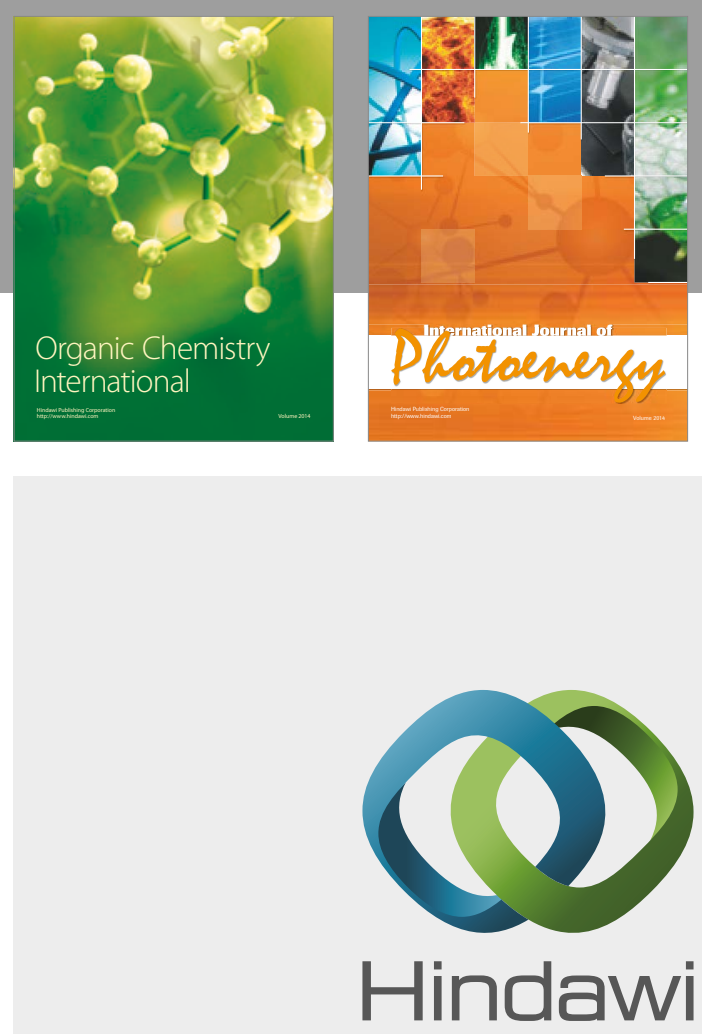

Submit your manuscripts at

https://www.hindawi.com

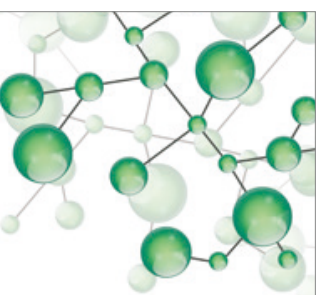

International Journal of

Inorganic Chemistry

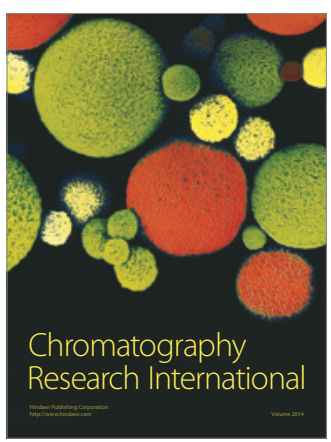

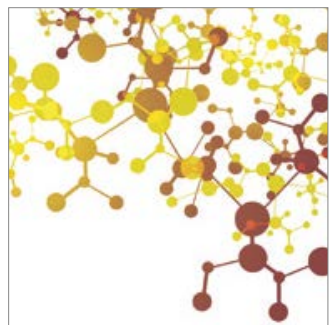

Applied Chemistry
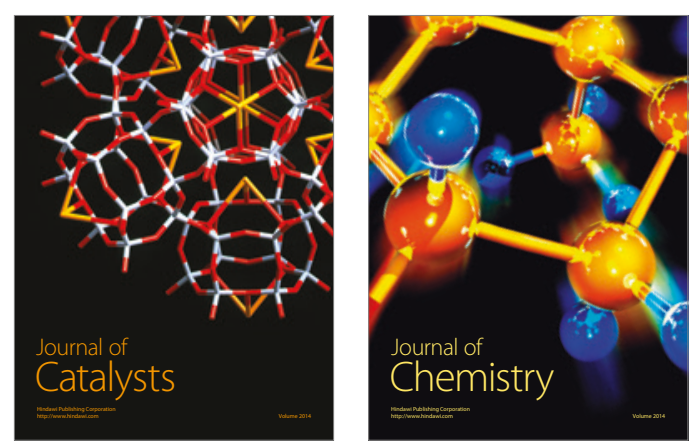
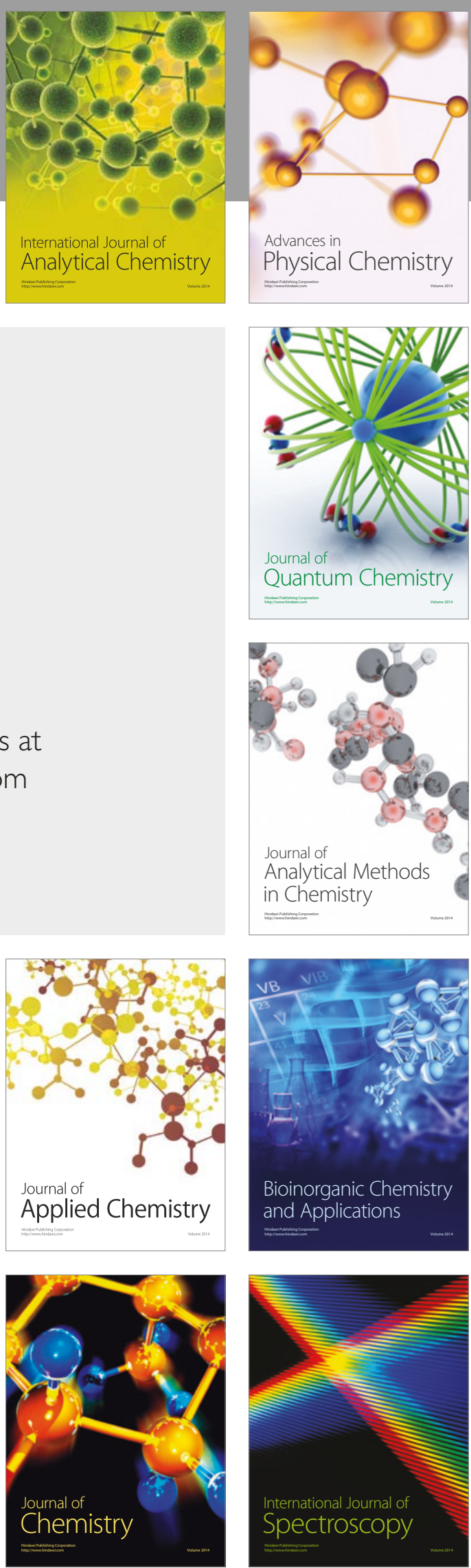